${ }^{1}$ Facultad de Enfermería, Universidad Andrés Bello. Santiago, Chile

${ }^{2}$ Asesoría independiente. Santiago, Chile.

${ }^{3}$ Departamento Gestión TIC, Servicio de Salud Metropolitano Sur. Santiago, Chile. ${ }^{a}$ Enfermero, Doctor ${ }^{\circledR}$ en Psicología

bEnfermera-matrona, Ingeniero Comercial, Doctora en Ciencias Empresariales.

'Estadístico, Licenciado en Matemáticas.

dIngeniero Civil Biomédico, Magister en Salud Pública especialista en Gestión Hospitalaria, MBA en Salud, Master en Salud

Pública especialista en Gestión Sanitaria.

Fuente de financiamiento: Proyecto de investigación interno Ciencias Biomédicas y Clínicas Universidad Andrés Bello, DI-04-19/CB, "Policonsultantes en Servicios de Atención Primaria de Salud: una aproximación mediante el uso de bases de datos y machine learning".

Comité de ética: Proyecto aprobado por el Comité Científico Ético del Servicio de Salud Metropolitano Sur, Memorandum 189/2019.

Los autores declaran no tener conflictos de interés.

Recibido el 28 de mayo de 2020, aceptado el 17 de septiembre de 2020

Correspondencia a: Katiuska Reynaldos-Grandón Avenida República 217. Santiago, Chile. katiuska.reynaldos@unab.cl

\section{Generalidades del Machine Learning y su aplicación en la gestión sanitaria en Servicios de Urgencia}

\author{
VÍCTOR PEDRERO ${ }^{1, \mathrm{a}}$, KATIUSKA REYNALDOS-GRANDÓN ${ }^{1, \mathrm{~b}}$, \\ JOAQUÍN URETA-ACHURRA ${ }^{2, \mathrm{c}}$, ERICK CORTEZ-PINTO ${ }^{3, \mathrm{D}}$
}

\section{Overview of machine learning and its application in the management of emergency services}

The processes associated with health care generate a large amount of information that is difficult to analyze using standard statistical procedures. In this context, disciplines such as Data Science became relevant, mainly through strategies such as Machine Learning (ML). The latter groups a series of tools whose purpose is to develop algorithms to extract information from data, whether for explanation, classification, or prediction. Despite its usefulness as support for clinical decisions, its potential in health care management has been less explored. Also, there are difficulties in understanding these types of studies. This work tries to offer a nontechnical overview of the ML concept and its advantages for health care management. It collects examples of ML applications in emergency department management.

(Rev Med Chile 2021; 149: 248-254)

Key words: Emergency Medical Services; Health Information Management; Machine Learning.
L os procesos involucrados en la atención de salud generan una gran cantidad de información que resulta difícil analizar. Esto se debe, entre otras cosas, al volumen, velocidad de producción y diversidad de los datos (por ejemplo: texto, imágenes, tiempos, registros administrativos $)^{1}$. Herramientas como el Machine Learning $\mathrm{u}$ otras técnicas de Data Science permiten lidiar con estas dificultades facilitando la entrega de información rápida y confiable que ayude a la toma de decisiones en las organizaciones de salud.

El Data Science, o Ciencia de los Datos, surge en la década de los sesenta y setenta al alero de la matemática y la estadística, como un tendencia que buscaba utilizar, mediante datos, el plan- teamiento de hipótesis, de modo de convertirlos en información y conocimiento ${ }^{2}$. Actualmente, la ciencia de los datos se define como un campo multidisciplinario que integra la estadística, informática, comunicación, sociología y administración para estudiar los datos, el entorno en que se producen y generar conocimiento que ayude a la toma de decisiones ${ }^{2}$.

Bajo el alero del Data Science conviven tanto las formas tradicionales de análisis de datos como nuevas aproximaciones ${ }^{2}$. Tradicionalmente los datos son analizados e interpretados a partir de hipótesis formuladas previamente. Las hipótesis conducen a seleccionar ciertas variables y poner aprueba determinadas relaciones entre ellas. Esta 
aproximación nos permite esclarecer, en parte, lo que está sucediendo en un set de datos particular y hacer ciertas predicciones. Sin embargo, para aprovechar al máximo la información contenida en grandes volúmenes de datos, como aquellos generados en la atención sanitaria, no es posible hipotetizar como cientos y a veces miles de variables se relacionan entre sí para explicar un determinado resultado ${ }^{2,3}$. Es por esto que se hace necesario encontrar nuevas formas de aproximarnos a los datos, especialmente cuando se desea desarrollar algoritmos predictivos que apoyen la toma de decisiones tanto clínicas como de gestión ${ }^{1,2}$. Uno de los recursos más populares en el campo del Data Science es el Machine Learning (ML) o aprendizaje automático. Este trabajo pretende ofrecer un panorama general sobre el concepto y utilidad para la gestión en salud del ML.

\section{Una aproximación general al aprendizaje automático o Machine Learning}

El ML es un término amplio que agrupa varias estrategias analíticas cuyo propósito es el desarrollo de algoritmos para extraer información de los datos ya sea para explicación, clasificación o predicción $n^{4,5}$. Pese a que se suele considerar como sinónimo de inteligencia artificial, es importante precisar que la inteligencia artificial es una clasificación aún más amplia que incluye tanto técnicas para el análisis de datos estructurados como el aprendizaje automático y datos no estructurados como procesamiento de lenguaje natural ${ }^{5}$. El ML surge en la década de los cincuenta como un recurso para emular, computacionalmente, elementos del proceso cognitivo humano a través de reconcomiendo de patrones y procesos de toma de decisión ${ }^{5}$. En el ámbito de la medicina ha sido utilizado para aumentar la precisión diagnóstica, hacer predicciones de mortalidad hospitalaria o predecir la necesidad de ciertas terapias ${ }^{5}$. Desde 2016 forma parte de los términos incluidos en el Medical Index Subheadings (MeSH) de Pubmed y ya es posible encontrar más de catorce mil artículos en esta materia.

Los algoritmos de ML pueden ser clasificados en: supervisados, no supervisados y parciamente supervisados ${ }^{5}$. La principal diferencia entre estos tres tipos de algoritmos es la presencia o ausencia de una variable de resultado o dependiente, a la cual se le denomina marca o etiqueta.

\section{Algoritmos no supervisados}

Los algoritmos no supervisados se caracterizan por su capacidad para extraer, clasificar y reducir la dimensionalidad. Este tipo de análisis es útil para la generación de nuevas hipótesis de trabajo ${ }^{4,5}$. Algunos ejemplos de este tipo algoritmos son: Cluster Analysis (o Análisis de Conglomerados) y Análisis de Componentes Principales (PCA, en inglés). El análisis de conglomerados permite agrupar unidades muestrales (por ejemplo, personas) de acuerdo con la similitud que existe entre ellas en un conjunto de variables numéricas. Por otro lado, el PCA es una técnica cuyo objetivo es la reducción de la dimensionalidad de los datos, su objetivo no es agrupar unidades muestrales, sino variables. Utilizando PCA, es posible reducir una gran cantidad de variables a un número menor de ellas sin perder demasiada información. Estos algoritmos no son excluyentes entre sí y podrían utilizarse en conjunto. Por ejemplo, Mesquita, Spina $^{6}$, utilizaron una estrategia que combinó PCA y análisis de conglomerados para identificar patrones de actividad física en pacientes con enfermedad pulmonar obstructiva crónica.

\section{Algoritmos supervisados}

El objetivo de los algoritmos supervisados es la predicción ${ }^{5}$. Para esto, el algoritmo es entrenado para relacionar un conjunto de variables con un resultado de interés. Una vez que el entrenamiento se ha completado, el algoritmo puede hacer predicciones utilizando nuevos datos ${ }^{5,7}$. Por ejemplo, se podrían utilizar para predecir el riesgo de contraer una enfermedad a partir de un conjunto de variables que se asocian a su aparición. El resultado de este tipo de análisis puede ser una clasificación (algoritmos de clasificación) así como también una probabilidad o un puntaje (algoritmo de predicción $)^{5}$. Algunos ejemplos de este tipo de algoritmos son el Support Vector Machine (SVM), Redes Neuronales, Ârboles de Decisión, Bosques Aleatorios, entre otros. Una revisión reciente mostró que el SVM y las Redes Neuronales están entre las técnicas más populares en el área de la salud ${ }^{5}$. El primero es un algoritmo de clasificación, mientras que el segundo puede ser utilizado tanto para clasificación como predicción. Un ejemplo del uso de redes neuronales es su aplicación es el análisis de imágenes para mejorar la precisión diagnóstica en pacientes con artritis reumatoide ${ }^{8}$. De la misma forma, algunos autores han propuesto el uso de 
SVM para apoyar el diagnóstico de enfermedad renal crónica9?.

\section{Algoritmos parcialmente supervisados}

Los algoritmos parcialmente supervisados comparten características de aquellos supervisados y no supervisados 5 . Una de sus ventajas es poder trabajar con datos en donde no todas las unidades cuentan con un valor en la variable resultado. Por ejemplo, un algoritmo supervisado puede ser entrenado con un subconjunto de los datos disponibles y luego aplicado para predecir los valores en la variable resultado en aquellos casos que no contaban con este valor. Un ejemplo de aplicación de este tipo de técnicas es el diagnóstico de cáncer de mama a partir de imágenes. Una dificultad de este proceso es que el periodo desde la toma de imagen hasta el desenlace del caso (sobrevida o no) puede tardar varios años e implicaría seguimiento de los pacientes. Para esto se han empleado algoritmos que permite utilizar los datos completos para predecir datos faltantes y calibrar el algoritmo con mayor cantidad de información ${ }^{10}$. El resultado es mayor precisión diagnóstica.

\section{Utilidad del Machine Learning en la gestión de salud}

Los algoritmos de aprendizaje automático, además de ser útiles para realizar predicciones clínicas y epidemiológicas, también pueden ser aplicados en la gestión de servicios de salud. En esta área, tienen el potencial de aportar al análisis de los procesos clínicos y administrativos que tienen lugar en una organización de salud ${ }^{11}$. Un proceso puede ser definido como una serie de pasos interconectados dentro de un contexto organizacional cuyo objetivo es crear bienes o servicios $^{12}$. Algunos ejemplos de procesos son la atención de un paciente en el servicio de urgencia, la gestión de insumos, la organización del personal sanitario, entre otros.

La evidencia ha mostrado que un aspecto necesario para entregar cuidados de salud de calidad es la integración de los servicios ${ }^{12}$. Esto implica integrar, no solo los diferentes procesos clínicos como podrían ser las atenciones de salud entregadas por diferentes especialistas, sino también procesos de carácter administrativo como la provisión de insumos o la gestión de costos. Estos procesos, usualmente no ocurren de forma lineal en una organización de salud sino que existen en paralelo e interactúan entre $s^{13}{ }^{13}$. Por lo tanto, intervenir un proceso determinado no solo impacta en su propio desempeño sino también en otros que tienen relación con él. La integración propone desafíos en el diseño, implementación y especialmente en la evaluación de los procesos que ocurren al interior de la organización de salud. Para todos estos aspectos es crucial analizar los datos derivados de tales procesos ${ }^{11}$.

Cada proceso de forma individual genera un gran volumen de datos en sus diferentes etapas. Por ejemplo, el proceso de atención en el servicio de urgencias genera datos durante el ingreso administrativo, en el categorizador de riesgo, en la atención médica, en el paso del paciente por el servicio de radiología, entre otros. La naturaleza de estos datos es diversa, algunos son más estructurados como por ejemplo los registros administrativos, mientras que otros tienen menos estructura como los registros clínicos o las imágenes. Para evaluar de forma efectiva un proceso como este y perfeccionarlo sería necesario analizar un gran volumen de datos de diferentes tipos $^{12}$. En este sentido las herramientas de ML son de gran utilidad ya que permiten analizar múltiples fuentes de datos con diferente nivel de estructura y no requieren hipótesis para dichos análisis. De esta forma el ML puede generar nuevo conocimiento que contribuye al aprendizaje organizacional, lo cual permite proporcionar, servicios más eficientes ${ }^{13}$.

El aprendizaje organizacional se refiere al proceso en el cual la organización considera información interna y externa a ella, para la toma de decisiones. Hoy en día, este aspecto se considera clave en función del logro de las metas, de cimentar la permanencia en el mercado y de trascender en él ${ }^{14}$. Las estrategias de ML podrían retroalimentar a la organización tanto a partir del procesamiento de su propia información así como también integrando información del entorno ${ }^{15,16}$. Ejemplo de ello es la Figura 1, que grafica el modelo de gestión del conocimiento utilizado por la Unidad de Ciencia de Datos del Servicio de Salud Metropolitano (SMSS), que considera el proceso de Dato, Información, Conocimiento y Acción, para entregarle al paciente una propuesta de estimación de tiempos de espera en las atenciones de urgencia que se realizan en los establecimientos de 
Figura 1. Modelo de Gestión del Conocimiento, Unidad de Ciencia de Datos del Servicio de Salud Metropolitano Sur. Autor: Enf. Natalia Gillies, Subjefa Departamento Gestión TIC SSMS.

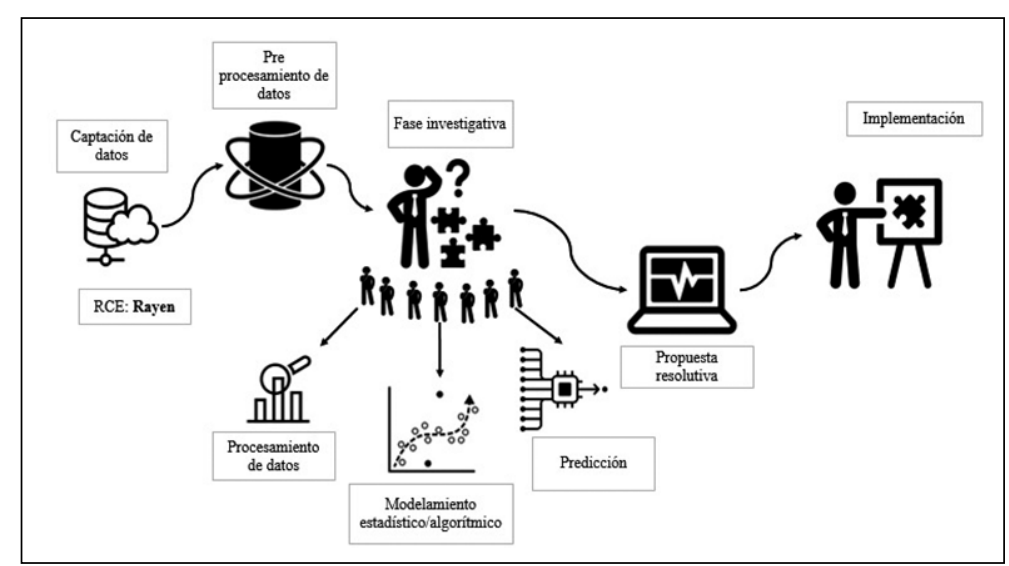

la red, donde la acción es definida por el mismo paciente: asistir, no asistir, donde asistir.

El ML optimiza la toma de decisiones e introduce mejoras en la prestación de servicios como los servicios de salud. Por lo tanto, aspectos como este deben ser parte de la agenda de innovación de cada institución sanitaria y ser capaces aprender, desaprender y volver a aprender ${ }^{14}$.

\section{Ejemplos uso de Machine Learning en la gestión de servicios de emergencia}

Dada las particularidades de Machine Learning, uno de sus usos en salud ha sido la elaboración de modelos que permitan establecer pronóstico ${ }^{17}$. Estos algoritmos tienen la capacidad de aprender las trayectorias de salud de los usuarios y en base a esto hacer predicciones de lo que les sucederá en el futuro ${ }^{17}$. Una de las áreas que se beneficia de este tipo de información son los servicios de emergencia. En esta área, el Machine Learning ha sido empleado para perfeccionar los procesos de triage ${ }^{18,19}$ así como también para identificar a aquellos pacientes que consultaran frecuentemente (policonsultantes) ${ }^{20}$. Ambas aplicaciones tienen importantes alcances tanto para la gestión de recursos humanos y materiales como para la toma de decisiones clínicas ${ }^{21}$.

\section{Aplicaciones en el triage}

El triage es proceso clasificación de riesgo que permite discriminar entre quienes requieren atención inmediata en el servicio de urgencia de quienes pueden esperar más tiempo. Al clasificar a los usuarios los profesionales de salud deben lidiar con al menos dos problemas subestimar o sobre estimar el riesgo ${ }^{18}$, es decir, asignar una mayor prioridad de atención a una persona con un situación de bajo riesgo vital o clasificar con un bajo riesgo a alguien que posee una condición crítica de salud. Los algoritmos de Machine Learning han demostrado ser útiles en esta área e incluso pueden presentar un mejor desempeño que los expertos ${ }^{19}$. Levin, Toerper ${ }^{19}$ compararon los resultados de un algoritmo de Machine Learning para triage con la clasificación de expertos. En este estudio, la precisión del algoritmo fue más alta que la de los expertos, especialmente en aquellas situaciones de emergencia clasificadas como moderadamente severas (ESI 3). Particularmente estos autores estimaron que el 10\% de este último grupo fue subcategorizado. En Chile, Wolff, Rios ${ }^{18}$ utilizando una base de datos de consultas de urgencia de tres años, bases de datos de los grupos relacionados al diagnóstico y datos administrativos, probaron distintos algoritmo de ML para realizar triage en un hospital de niños. Los resultados mostraron que algunos de los algoritmos examinados eran más precisos que el juicio experto.

\section{Identificación de pacientes policonsultantes}

En los servicios de urgencia los pacientes policonsultantes o hiperfrecuentadores son responsables de hasta un $30 \%$ del total de consultas en estos servicios $^{22}$. El fenómeno de la policonsulta puede ser explicado tanto por variables propias de los 


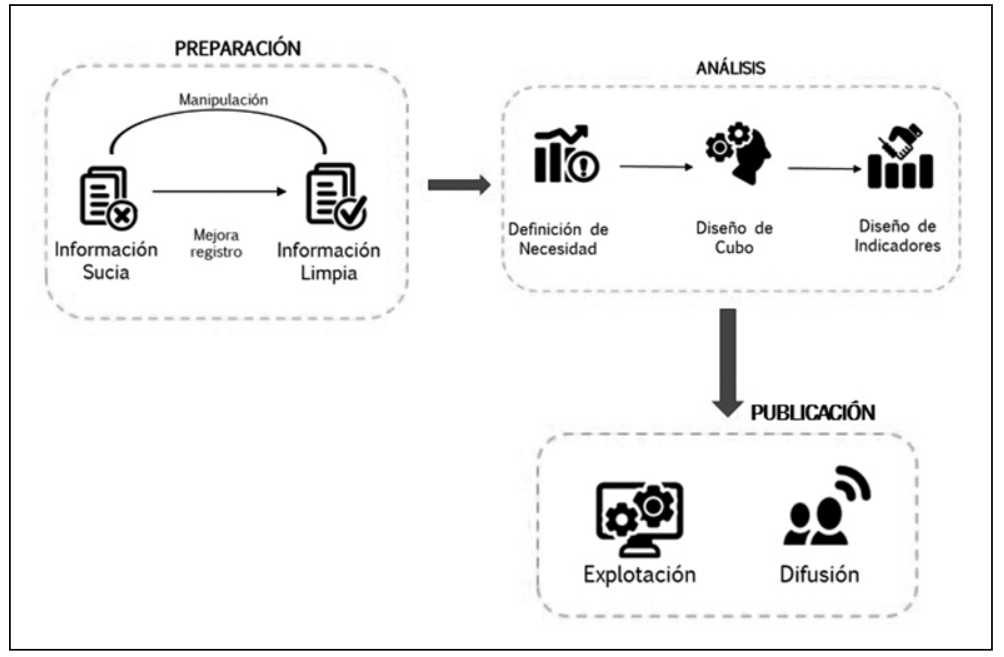

Figura 2. Esquema general de las etapas de la aplicación de ML para detectar usuarios potencialmente policonsulantes en servicios de urgencia. Autor: Elaboración propia. usuarios (por ejemplo, la edad de los usuarios, el grado de vulnerabilidad, la presencia de patologías psiquiátricas, entre otros) como del sistema de atención (por ejemplo, baja resolutividad de los servicios, problemas de dotación de personal ${ }^{23}$. El Machine Learning ha sido propuesta como una estrategia efectiva para identificar pacientes policonsultantes ${ }^{20,24}$. Identificar a estos pacientes permite diseñar intervenciones apropiadas para abordar sus necesidades específicas de salud ${ }^{25}$. Por ejemplo, Mesgarpour, Chaussalet ${ }^{20}$, utilizaron datos administrativos, clínicos y características de los paciente en Inglaterra para elaborar un algoritmo de ML para predecir readmisiones en el servicio de urgencia con buenos resultados. En Chile existe escasa información sobre los usuarios policonsultantes. Se estima que entre el 10 y $30 \%$ de las consultas en atención primaria corresponden a este tipo de pacientes ${ }^{26}$. A continuación, se describe el diseño de una investigación en curso que utiliza ML para identificar pacientes policonsultantes en servicio de urgencia de APS. Este ejemplo, esquematizado en la Figura 2, clarifica las diferentes etapas del proceso considerando la relevancia que tiene el desarrollo de algoritmos adecuados a la realidad local ${ }^{18}$.

\section{Etapa 1. Adquisición de datos}

El primer paso es la recopilación de información a partir de diversos sistemas de información de salud disponibles, por ejemplo, del Registro
Clínico Electrónico (RCE) Rayén. Esta información se podría complementar, con otras fuentes de datos como, encuestas de salud pública, información contenida en medios de comunicación (tanto escrito como no escrito), entre otras.

\section{Etapa 2. Procesamiento de datos}

En esta etapa se evalúa la disponibilidad e integridad de los datos y la presencia de datos perdidos (o ausentes). Además, se cautela la privacidad, anonimización y confidencialidad de la información. Esta etapa es crítica y de alto aliento, puesto que información de mala calidad en especial, en los registros de los textos libres, podría generar conclusiones incorrectas. Dentro de los problemas que se pueden presentar en los datos se encuentran: irregularidades numéricas, categorizaciones incorrectas, existencia de caracteres no numéricos en variables numéricas, ausencia de registros, entre otros. En base a estos posibles problemas, se deben definir criterios de exclusión de registros, así como considerar técnicas específicas de corrección de tales problemas.

\section{Etapa 3. Exploración de datos}

En esta etapa se obtienen los primeros resultados sobre el fenómeno de estudio. Sin embargo, no permite generar predicciones sobre el comportamiento futuro de los integrantes de la muestra. Con el fin de caracterizar la muestra en estudio se pueden comparar los datos con marcas de policon- 
sultante $\mathrm{v} / \mathrm{s}$ no policonsultante utilizando técnicas de resumen de datos y visualización (histogramas, boxplots, estadísticos de resumen, ANOVA).

\section{Etapa 4. Análisis estadístico y modelamiento de datos}

En esta etapa se explora el comportamiento predictivo diversos métodos tanto estadísticos como algorítmicos. Es aquí, donde ML entrega una batería de alternativas para evaluar supuestos con sustento estadístico, proponer métodos explicativos sólidos, y generar predicciones. En el caso de la detección de pacientes policonsultantes, dado la información de la variable de interés (número de asistencias a servicios de salud) se encuentra disponible en las bases de datos, se pueden utilizar algoritmos supervisados. Al finalizar la etapa, el grupo de investigación estará en posición de proponer una solución consistente para responder la (o las) pregunta de investigación propuesta.

\section{Etapa 5. Validación y selección del modelo}

Para realizar la validación y elección final del modelo, se deben considerar ciertas características asociadas a su desempeño entre ellas: su calidad predictiva y nivel de generalización. Para medir la calidad predictiva se pueden utilizar métricas provenientes de la matriz de confusión (tabla de contingencia), en conjunto con la curva ROC. Para la generalización del modelo una alternativa es aplicar del modelo a una nueva muestra integrada por individuos que el modelo, en su proceso de entrenamiento, no ha observado. Por ejemplo, otra muestra de pacientes que asisten a un servicio de urgencia.

Para definir umbrales de aprobación del modelo se debe tener presente la incidencia de consultantes en la población de estudio. No siempre es posible definir umbrales a priori y es aconsejable considerar la opinión de expertos en salud.

\section{Etapa 6. Representación y reporte}

Finalmente se operacionaliza la solución de manera funcional, por ejemplo, a través de un software. El empaquetamiento permitiría utilizar el modelo, o algoritmo, y en tiempo real, para predecir el comportamiento de un nuevo paciente categorizándolo como policonsultante o no. Además, hace posible evaluar escenarios simulados lo cual contribuye apoyando la toma de decisiones activas.

\section{Conclusiones}

La atención de salud genera un considerable volumen de datos, los cuales son difíciles de analizar con las técnicas estadísticas convencionales. En este sentido, las estrategias basadas en ciencia de datos y particularmente en ML resultan un recurso útil. Si bien estas aproximaciones han sido más utilizadas en el ámbito del diagnóstico clínico también representan una oportunidad para mejorar la gestión asistencial en las organizaciones de salud. Algunos ejemplos ilustrados en este trabajo son sus aplicaciones en la clasificación de pacientes y en la detección de usuarios policonsultantes. Actualmente, las bases de datos disponibles en nuestro país, como son, aquellas provenientes de los registros electrónicos e incluso de las imágenes almacenadas, ofrecen una importante oportunidad para generar conocimiento a partir de los datos existentes y retroalimentar a las organizaciones de salud contribuyendo a la toma de decisiones.

Agradecimientos: A Natalia Gillies, enfermera, integrante del equipo del Departamento Gestión TIC del SSMS.

\section{Referencias}

1. van der Aalst W. Process Mining. Berlin, Heidelberg: Springer Berlin Heidelberg; 2016.

2. Cao L. Data Science: A Comprehensive overview. ACM Computing Surveys. 2017; 50 (3): 1-42.

3. Beam AL, Kohane IS. Big Data and machine learning in health care. JAMA. 2018; 319 (13): 1317.

4. Wiemken TL, Kelley RR. Machine learning in epidemiology and health outcomes research. Annual Review of Public Health. 2020; 41 (1): 21-36.

5. Jiang F, Jiang Y, Zhi H, Dong Y, Li H, Ma S, et al. Artificial intelligence in healthcare: past, present and future. Stroke and Vascular Neurology. 2017; 2 (4): 230-43.

6. Mesquita R, Spina G, Pitta F, Donaire-Gonzalez D, Deering BM, Patel MS, et al. Physical activity patterns and clusters in 1001 patients with COPD. Chron Respir Dis. 2017; 14 (3): 256-69.

7. Sidey-Gibbons JAM, Sidey-Gibbons CJ. Machine learning in medicine: a practical introduction. BMC Medical Research Methodology. 2019; 19 (1): 64.

8. Andersen JKH, Pedersen JS, Laursen MS, Holtz K, Grauslund J, Savarimuthu TR, et al. Neural networks 
for automatic scoring of arthritis disease activity on ultrasound images. RMD open. 2019;5(1):e000891.

9. Polat H, Mehr HD, Cetin A. Diagnosis of chronic kidney disease based on support vector machine by feature selection methods. J. Med. Syst. 2017; 41 (4): 55.

10. Zemmal N, Azizi N, Dey N, Sellami M. Adaptive semi supervised support vector machine semi supervised learning with features cooperation for breast cancer classification. J. Med. Imaging \& Health Infor. 2016; 6 (1): 53-62.

11. Rojas E, Muñoz-Gama J, Sepúlveda M, Capurro D. Process mining in healthcare: A literature review. J Biomed Inform. 2016; 61: 224-36.

12. Buttigieg SC, Dey PK, Gauci D. Business process management in health care: current challenges and future prospects. Innovation and Entrepreneurship in Health. 2016; 3: 1-13.

13. Mora J. Gestión por Precesos: Conceptos, Fundamentos y Enfoque Metodológico. 1a ed. Madrid, España: José Ramón Mora Martínez; 2016.

14. Mora G. Siglo XXI economía de la información: gestión del conocimiento y Business Intelligence, el camino a seguir hacia la competitividad. Signos. 2018; 10 (2): 161-74.

15. Bailetti T, Gad M, Shah A. Intrusion learning: an overview of an emergent discipline. Technol. Innov. Manag. Rev. 2016; 6 (2): 15-20.

16. Garad AKA, Gold J. The learning-driven organization: Towards an integrative model for organizational learning. Ind. Commer. Train. 2019; 51 (6): 329-41.

17. Rajkomar A, Dean J, Kohane I. Machine learning in medicine. N. Engl. J. Med. 2019; 380 (14): 1347-58.

18. Wolff P, Rios SA, Grana M. Setting up standards: A methodological proposal for pediatric Triage machine learning model construction based on clinical outcomes. Expert Syst Appl. 2019; 138:UNSP-112788.

19. Levin S, Toerper M, Hamrock E, Hinson JS, Barnes S, Gardner H, et al. Machine-learning-based electronic triage more accurately differentiates patients with respect to clinical outcomes compared with the Emergency Severity Index. Ann Emerg Med. 2018; 71 (5): 565-74.

20. Mesgarpour M, Chaussalet T, Chahed S. Ensemble Risk Model of Emergency Admissions (ERMER). Int J Med Inform. 2017; 103: 65-77.

21. Bates DW, Saria S, Ohno-Machado L, Shah A, Escobar G. Big data in health care: using analytics to identify and manage high-risk and high-cost patients. Health Aff. 2014; 33 (7): 1123-31.

22. Matsumoto CL, O'Driscoll T, Madden S, Blakelock B, Lawrance J, Kelly L. Defining" high-frequency" emergency department use: Does one size fit all for urban and rural areas? Can Fam Physician. 2017; 63 (9): e395-e9.

23. Reynaldos-Grandón K, Nasabún-Flores V, Pedrero-Castillo V, Cortéz-Pinto E, Ureta-Achurra J, Pavlov-Sepúlveda T. Pacientes policonsultantes: variabilidad de concepto. Rev Med Chil. 2020; 148 (1): 128-9.

24. Besga A, Ayerdi B, Alcalde G, Manzano A, Lopetegui P, Graña $M$, et al. Risk factors for emergency department short time readmission in stratified population. Biomed Res Int. 2015; 2015: 1-7.

25. Smits FT, Wittkampf KA, Schene AH, Bindels PJ, van Weert HC. Interventions on frequent attenders in primary care. Scand J Prim Health Care. 2008; 26 (2): 111-6.

26. Miranda Hiriart G, Saffie Gatica X. Pacientes policonsultantes: ¿un síntoma del sistema de salud en Chile? Acta Bioeth. 2014; 20 (2): 215-23. 